ARTIGO ORIGINAL ORIGINAL ARTICLE
Palavras-chave:

análise de custo-efetividade, neoplasias da próstata, acetato de abiraterona

\section{Análise de custo-efetividade do acetato de abiraterona associado à prednisona pós-terapia de privação androgênica, seguido de enzalutamida pós-quimioterapia versus a sequência oposta para o tratamento de pacientes com câncer de próstata metastático resistentes à castração sob a perspectiva do Sistema de Saúde Suplementar brasileiro}

\author{
Cost-effectiveness analysis of abiraterone acetate plus \\ prednisone in the post-androgen deprivation therapy setting \\ followed by enzalutamide in the post-chemotherapy setting \\ versus the opposite treatment sequence in metastatic \\ castration resistant prostate cancer patients under the \\ Brazilian Private Healthcare System perspective
}

Lilian Pititto', Elio Asano'

DOI: 10.21115/JBES.v9.n1.p83-92

\section{RESUMO}

Objetivo: $O$ acetato de abiraterona e a enzalutamida são utilizados no tratamento de câncer de próstata metastático pós-terapia de privação androgênica, em pacientes resistentes à castração. 0 objetivo deste estudo foi comparar o custo-efetividade de abiraterona mais prednisona pós-terapia de privação androgênica, seguidos de docetaxel e enzalutamida pós-quimioterapia com a sequência oposta de tratamento no Sistema de Saúde Suplementar brasileiro. Métodos: Um modelo de Markov foi desenvolvido para comparar o custo-efetividade das duas sequências em um tempo horizonte lifetime. Os parâmetros de eficácia e probabilidades de transição foram derivados de estudos clínicos. Foram considerados os custos diretos dos medicamentos, administração, monitoramento e eventos adversos. A medida de efetividade foram anos de vida ganhos, estimados pela extrapolação de dados dos estudos clínicos. Os resultados foram apresentados em custos e anos de vida ganho a cada sequência. Resultados: $\bigcirc$ estado pós-terapia de privação androgênica representou a maior parte dos custos de tratamento, e os eventos adversos tiveram pequeno impacto nos custos totais. O uso de abiraterona nesse estado reduziu 7,3\% dos custos. A sequência abiraterona mais prednisona pós-terapia de privação androgênica, seguida de enzalutamida pós-quimioterapia, foi dominante em relação à oposta; apresentou menor custo ( $R \$ 262.801$ versus $R \$ 274.165)$ e efetividade levemente maior, com estimados 3,367 anos de vida ganhos versus 3,282. Conclusão: $\bigcirc$ uso da abiraterona mais prednisona pós-terapia de privação androgênica e enzalutamida pós-quimioterapia demonstrou-se dominante em relação à sequência oposta no tratamento de pacientes com câncer de próstata metastático resistentes à castração, no Sistema de Saúde Suplementar brasileiro. 


\section{Keywords:}

cost-effectiveness analysis, prostatic neoplasms,

abiraterone acetate

\begin{abstract}
Objective: Abiraterone acetate and enzalutamide are important options in the treatment of metastatic castration resistant prostate cancer. The objective of this study is to compare the cost-effectiveness of the use of abiraterone plus prednisone post-ADT, followed by docetaxel and enzalutamide post-chemotherapy (Abi+Doce+Enza) with the opposite treatment sequence (Enza+Doce+Abi) under the perspective of the Brazilian private healthcare system. Methods: A Markov model was developed to assess the cost-effectiveness of both sequences in a lifetime time horizon. Transition probabilities and efficacy data were drawn from clinical studies. Cost parameters included drug acquisition and administration, disease monitoring and adverse events were considered. Effectiveness was measured as life years gained, derived from clinical trials. Results were presented as total costs and life years gained in each sequence. Results: Post-ADT state represented the majority of the treatment costs, and adverse events had little impact in total costs. The sequence Enza+Doce+Abi was dominated by Abi+Doce+Enza. The sequence Abi+Doce+Enza generated a reduction of 7,3\% in total treatment costs compared to Enza+Doce+Abi (R\$262,801 vs R\$274,165). Effectiveness was slightly higher, with an estimated 3.367 life-years gained in the Abi+Doce+Enza sequence compared with 3.282 life-years gained in the Enza+Doce+Abi sequence. Conclusion: The use of abiraterone plus prednisone post-ADT and enzalutamide in post-chemo had lower treatment costs and higher effectiveness when compared to the opposite sequence in the treatment of metastatic castration resistant prostate cancer, under the Brazilian Private Health System perspective.
\end{abstract}

\section{Introdução}

O câncer da próstata é a neoplasia mais frequente entre os homens de idade avançada no Reino Unido, Estados Unidos e Europa Ocidental (Tunn, 2007). Estima-se que a incidência de câncer de próstata no Brasil seja de 61,82 novos casos a cada 100 mil homens (Inca, 2015). Até 40\% dos casos diagnosticados eventualmente desenvolverão doença metastática (Beltran et al., 2011; Botrel et al., 2012). Para os pacientes com tumores avançados, recorrentes ou metastáticos, o bloqueio androgênico constitui, na maioria dos casos, a primeira linha de tratamento; desses pacientes, muitos evoluem para o estado resistente à castração (CPRC), cuja sobrevida global média varia de dois a três anos (Beltran et al., 2011; Botrel et al., 2012; Cha \& Fong, 2010; Chang \& Kibel, 2009; Tunn, 2007).

A mitoxantrona foi a primeira medicação quimioterápica aprovada, em 1996, para o tratamento de CPRC, baseada em dados de aumento da qualidade de vida dos pacientes com CPRC (Tannock et al., 1996). O docetaxel foi aprovado pelo Food and Drug Administration (FDA) em 2004, após dois estudos de fase III evidenciarem vantagens na sobrevida de pacientes com CPRC, e foi estabelecido como primeira linha de tratamento (Bianchini et al., 2010; Petrylak et al., 2004; Tannock et al., 1996). Desde então, novas tecnologias como a abiraterona (de Bono et al., 2011; Scher et al., 2011) e a enzalutamida (Scher et al., 2012) foram desenvolvidas e introduzidas no mercado, inicialmente após falha do docetaxel e depois em pacientes ainda não expostos ao regime quimioterápico (Beer et al., 2014; Ryan et al., 2013).

A abiraterona é um inibidor seletivo da biossíntese androgênica, inibidor do citocromo P450 c17 (CYP17), crucial para a síntese de testosterona (Attard et al., 2005). Dois estudos de fase III demonstraram que a abiraterona apresentou ga- nho de sobrevida comparada ao placebo no tratamento de pacientes com CPRC, e sua aplicação antes do regime com docetaxel resultou em sobrevida maior do que o uso após o regime quimioterápico (de Bono et al., 2011; Fizazi et al., 2012). Os estudos de fase III que compararam o uso da enzalutamida com placebo no tratamento de CPRC também demonstraram maior ganho de sobrevida global com o seu uso após a falha do docetaxel (Beer et al., 2014; Scher et al., 2012).

A melhor sequência de tratamento para CPRC ainda é controversa e um dos maiores desafios para a prática médica (Sadi et al., 2011), uma vez que estudos clínicos sugeriram que o uso de abiraterona e enzalutamida antes de docetaxel pode aumentar a sobrevida global dos pacientes com CPRC (Beer et al., 2014; Ryan et al., 2013).

O objetivo do presente estudo foi avaliar o custo-efetividade de duas sequências terapêuticas utilizadas para o tratamento de CPRC: abiraterona associada à prednisona, seguida de docetaxel e então enzalutamida (AAP-DOC-ENZ) e enzalutamida seguida de docetaxel e então abiraterona associada à prednisona (ENZ-DOC-AAP), sob a perspectiva do Sistema de Saúde Suplementar brasileiro.

\section{Métodos}

\section{Racional clínico}

Quatro estudos clínicos internacionais, multicêntricos, randomizados, duplos-cegos e controlados por placebo foram selecionados para o desenvolvimento do racional clínico desta análise de custo-efetividade por meio de uma revisão de literatura e por serem os estudos pivotais que embasaram a aprovação regulatória do acetato de abiraterona e da enzalutamida. Os quatro estudos consideraram sobrevida global (SG) como desfecho primário de eficácia e relataram a 
duração mediana do tratamento até o momento da análise, definido como o intervalo entre a primeira dose e a descontinuação do medicamento (Beer et al., 2014; Fizazi et al., 2012; Ryan et al., 2015; Scher et al., 2012).

O estudo COU-AA-301 avaliou a eficácia e a segurança de abiraterona associada à prednisona, comparada com placebo associado à prednisona, em 1.195 pacientes com câncer de próstata após a progressão e previamente tratados com docetaxel. A SG mediana do grupo abiraterona foi de 15,8 meses [interquartile range (IQR): 14,8-17,0), enquanto no grupo placebo foi de 11,2 meses (IQR: 10,4-13,1), o que representa uma redução de $26 \%$ do risco de morte nos pacientes do grupo abiraterona [hazard ratio (HR) = 0,74 (IC 95\%: 0,64-0,86; $\mathrm{p}<0,0001)$ ]. A mediana de tempo de tratamento com abiraterona foi de 7,4 meses (IQR: 0,2-25,6) (Fizazi et al., 2012).

No estudo COU-AA-302, 1.088 pacientes com câncer de próstata metastático sem prévia exposição à quimioterapia foram randomizados para grupos de tratamento com abiraterona ou placebo, ambos associados à prednisona. A mediana de SG para esse grupo foi de 34,7 meses (IQR: 32,7-36,8), enquanto no grupo placebo foi de 30,3 meses (IQR: 28,7-33,3), totalizando uma redução de $19 \%$ no risco de morte para o grupo abiraterona [HR =0,81 (IC 95\%: 0,70-0,93; $p=0,0033$ )]. A mediana do tempo de tratamento do grupo abiraterona foi de 13,8 meses (IQR: 8,3-27,4) (Ryan et al., 2015).

A eficácia de enzalutamida para o tratamento de pacientes com câncer de próstata metastático resistentes à castração (CPRC) foi avaliada em dois estudos clínicos de fase III controlados por placebo (Beer et al., 2014; Scher et al., 2012).

No estudo AFFIRM, foram analisados 1.199 pacientes previamente tratados com docetaxel. A mediana de SG desses pacientes foi de 18,4 meses (IQR: 17,3 - não atingido), comparados a 13,6 meses (IQR: 11,3-15,8) no grupo placebo. A redução do risco de morte no grupo enzalutamida foi de 37\% $[H R=0,63$ (IC 95\%: 0,53-0,75; $p<0,001)]$. A mediana do tempo de tratamento com enzalutamida foi de 8,3 meses (Scher et al., 2012).

O estudo PREVAIL randomizou 1.717 pacientes que não haviam sido previamente expostos à quimioterapia. A comparação dos grupos apresentou redução de $29 \%$ no risco de morte dos pacientes do grupo enzalutamida $[H R=0,71$ (IC 95\%: 0,60-0,84; $p<0,001$ )], sendo as medianas de SG dos grupos tratamento e controle de 32,4 e 30,2 meses, respectivamente. Uma análise interina previamente planejada apresentou a mediana de tempo de tratamento com enzalutamida de 16,6 meses (Beer et al., 2014).

Tanto a abiraterona quanto a enzalutamida mostraram-se mais eficazes do que o placebo para o tratamento de pacientes com CPRC previamente expostos à quimioterapia ou não. Entretanto, nota-se uma diferença de SG para o mesmo medicamento, quando utilizado em momentos diferentes do tratamento. A SG dos estudos que analisaram a abiraterona e a enzalutamida antes da quimioterapia foi maior do que a SG dos estudos que analisaram o uso posterior. Além disso, a SG da abiraterona administrada pós-terapia de privação androgênica (pós-ADT) foi ainda maior do que a SG dos pacientes que receberam enzalutamida pós-ADT (Beer et al., 2014; Fizazi et al., 2012; Ryan et al., 2015; Scher et al., 2012).

Os critérios de inclusão estabelecidos para seleção de pacientes nesses quatro estudos clínicos foram similares. Portanto, na ausência de estudos clínicos de comparação direta entre as duas diferentes formas de sequenciamento da abiraterona e da enzalutamida, realizou-se uma matching adjusted indirect treatment comparison (MAIC) dos dados dos estudos PREVAIL e COU-AA-302. Assim, podem-se combinar dados das populações desses estudos e permitiu-se a comparação dos seus resultados (Beer et al., 2014; Signorovitch, 2012; Ryan et al., 2015).

Com base nesse racional, a presente análise de custo-efetividade vai comparar duas opções de sequenciamento dessas terapias - AAP-DOC-ENZ e ENZ-DOC-AAP - para o tratamento do CPRC.

\section{Análise de custo-efetividade (ACE)}

Uma vez que os desfechos clínicos foram diferentes nos estudos de abiraterona e enzalutamida, antes e após quimioterapia com docetaxel, optou-se por desenvolver uma análise de custo-efetividade para comparar custos e consequências de diferentes sequenciamentos desses medicamentos no tratamento de CPRC. Nesse tipo de análise, os benefícios clínicos são aferidos em unidades naturais. No presente estudo, as medidas utilizadas foram anos de vida ganhos (AVG) (Brasil, 2014).

\section{Perfil dos pacientes}

Esta ACE considerou como população-alvo pacientes com câncer de próstata metastático e resistentes à castração após terapia de privação androgênica e não previamente expostos a regime quimioterápico.

\section{Perspectiva}

Adotou-se a perspectiva do Sistema de Saúde Suplementar brasileiro.

\section{Tempo horizonte}

Considerou-se um tempo horizonte de 20 anos (lifetime), para permitir mensurar na totalidade a efetividade (anos de vida ganho) de ambos os comparadores.

\section{Modelo}

Um modelo de Markov estruturado para a simulação do curso clínico dos pacientes com CPRC sob a perspectiva do Reino Unido foi adaptado à realidade brasileira (Dearden, 2015). Posto que o objetivo do estudo foi comparar o uso de 
abiraterona e enzalutamida antes e depois de quimioterapia, as etapas do tratamento foram divididas em sete estados (Figura 1).

Todos os pacientes entram no modelo no estado de tratamento após terapia de privação androgênica, no qual podem ser tratados com abiraterona mais prednisona ou enzalutamida, dependendo da sequência comparadora utilizada. Os pacientes que descontinuam o tratamento migram então para o estado de monitoramento até que se inicie o tratamento quimioterápico.

No estado de tratamento quimioterápico, o regime adotado foi docetaxel associado à prednisona. Pacientes em risco de descontinuação migram para um novo estado de monitoramento, do qual eles podem migrar para o tratamento pós-quimioterapia.

No estado de tratamento pós-quimioterapia, os pacientes podem ser tratados com abiraterona mais prednisona ou enzalutamida, de acordo com a sequência comparadora utilizada. Assumiu-se que os pacientes que descontinuam o tratamento migram para o estado de cuidado paliativo. Os pacientes de todos os estados estão sujeitos a migrar para o estado de morte.

Foram definidos ciclos mensais de tratamento.

\section{Parâmetros}

\section{Parâmetros do modelo}

Nos estados pré-quimioterápicos (tratamento pós-ADT e monitoramento), os parâmetros utilizados foram extraídos dos estudos COU-AA-302, para a sequência AAP-DOC-ENZ, e PREVAIL, para a sequência ENZ-DOC-AAP. A mortalidade pós-ADT, o tempo para quimioterapia e o tempo de tratamento de ambas as sequências foram extrapolados por meio do

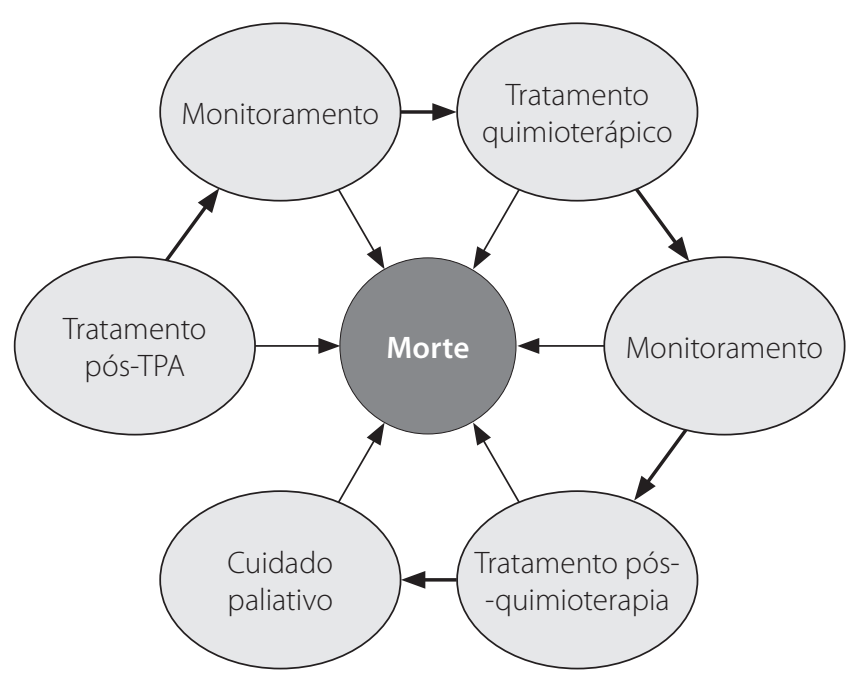

TPA: terapia de privação androgênica.

Figura 1. Modelo de Markov. método de regressão de Weilbull para que fossem analisados a longo prazo.

Os dados utilizados no estado de tratamento quimioterápico foram extraídos do estudo clínico de fase III, internacional, randomizado e multicêntrico, que compara o docetaxel com a mitoxantrona para o tratamento de pacientes com adenocarcinoma de próstata metastático. A mediana de tempo de tratamento foi de 6,6 meses, calculada com base na mediana do número de ciclos apresentada no estudo [9,5 (IQR: 1,0-11,0)] para o regime de docetaxel administrado a cada 21 dias. A mediana de SG foi de 18,9 meses (IQR: 17,0-21,2) e, com base nela, foi definido o risco de morte para esse estado e para o estado de monitoramento (Tannock et al., 2004).

No estado de tratamento pós-quimioterápico, os dados de tempo de tratamento e sobrevida global dos estudos COU-AA-301 e AFFIRM, conforme descrito na seção "Racional clínico", foram utilizados para estimar as probabilidades de transição e proporção de pacientes que permaneceram nesse estado e migraram dele para a morte ou cuidado paliativo a cada ciclo (Fizazi et al.; 2012, Scher et al., 2012)

Assumiu-se que a probabilidade de morte e o tempo de duração do estado de cuidado paliativo fossem baseados na SG dos pacientes que receberam placebo mais prednisona no estudo COU-AA-301 [11,2 meses (IQR: 10,4-13,1)] (Fizazi et al., 2012).

\section{Probabilidades de transição}

As probabilidades de transição entre os estados pós-ADT foram estimadas por meio das extrapolações dos dados de SG, tempo para início da quimioterapia e duração de tratamento dos estudos COU-AA-302 e PREVAIL, realizadas por Weibull (Tabela 1).

Para o estado de tratamento quimioterápico, partiu-se da mediana de duração de tratamento e calculou-se o hazard rate mensal de o paciente finalizar o tratamento quimioterápico e ingressar no estado de monitoramento. Do hazard rate, estimou-se a probabilidade de transição entre esses dois estados, por meio das fórmulas abaixo. O mesmo foi feito a partir da mediana de SG dos pacientes com docetaxel para estimar a probabilidade de transição para o estado de morte (Tabela 1) (Tannock et al., 2004).

hazard rate $=\frac{\ln 2}{\text { Mediana de tempo para evento }}$

Probabilidade de transição $=1-\mathrm{c}^{\text {hazard rate }}$

Do estado de monitoramento para o estado de tratamento pós-quimioterapia, estimou-se a probabilidade de transição a partir do resultado da subtração entre o tempo mediano até o início do regime pós-quimioterapia e o tem- 
Tabela 1. Probabilidades de transição

\begin{tabular}{|c|c|c|c|c|c|}
\hline \multicolumn{2}{|c|}{ Transição } & \multirow{2}{*}{$\begin{array}{l}\text { Tempo mediano } \\
\text { para o evento } \\
\text { (meses) }\end{array}$} & \multirow[b]{2}{*}{ Hazard rate (mensal) } & \multirow{2}{*}{$\begin{array}{l}\text { Probabilidade } \\
\text { (mensal) }\end{array}$} & \multirow[b]{2}{*}{ Fonte } \\
\hline $\mathrm{De}$ & Para & & & & \\
\hline TQ & Monit. & $6,6^{*}$ & 0,105 & 0,100 & Tannock et al. \\
\hline TQ & Morte & $18,9^{* *}$ & 0,037 & 0,036 & Tannock et al. \\
\hline Monit. & TPQ & $7,0^{*}$ & 0,099 & 0,094 & AFFIRM e Tannock et al. \\
\hline Monit. & Morte & - & - & 0,036 & Tannock et al. \\
\hline \multicolumn{6}{|c|}{ Abiraterona + Prednisona } \\
\hline P-ADT & Monit. & - & - & 0,0014 & COU-AA-302 (Weilbull) \\
\hline P-ADT & TQ & - & - & 0,0070 & COU-AA-302 (Weilbull) \\
\hline P-ADT & Morte & - & - & 0,0237 & COU-AA-302 (Weilbull) \\
\hline TPQ & $C P$ & $7,4^{*}$ & 0,094 & 0,089 & COU-AA-301 \\
\hline TPQ & Morte & $15,8^{* *}$ & 0,044 & 0,043 & COU-AA-301 \\
\hline$C P$ & Morte & $11,2^{* *}$ & 0,062 & 0,060 & COU-AA-301 \\
\hline \multicolumn{6}{|c|}{ Enzalutamida } \\
\hline P-ADT & Monit. & - & - & 0,0014 & PREVAIL (Weilbull) \\
\hline P-ADT & TQ & - & - & 0,0021 & PREVAIL (Weilbull) \\
\hline P-ADT & Morte & - & - & 0,0197 & PREVAIL (Weilbull) \\
\hline TPQ & $C P$ & $8,3^{*}$ & 0,084 & 0,080 & AFFIRM \\
\hline TPQ & Morte & $18,4^{* *}$ & 0,038 & 0,037 & AFFIRM \\
\hline$C P$ & Morte & $11,2^{* *}$ & 0,062 & 0,060 & COU-AA-301 \\
\hline
\end{tabular}

TQ: tratamento quimioterápico; Monit.: monitoramento; P-ADT: pós-terapia de privação androgênica; TPQ: tratamento pós-quimioterapia; CP: cuidado paliativo.

* Evento: fim do tratamento (mediana de tempo de tratamento).

** Evento: morte (mediana de sobrevida global).

po mediano de tratamento com docetaxel (Fizazi et al., 2012; Scher et al., 2012; Tannock et al., 2004). Assumiu-se que a probabilidade de transição entre o estado de monitoramento e morte fosse igual à probabilidade entre o estado de quimioterapia e morte (Tabela 1).

O mesmo método foi utilizado para calcular as probabilidades de transição entre o estado de tratamento pós-quimioterapia e os estados de cuidado paliativo e morte, para cada comparador. Para a transição entre o estado de cuidado paliativo e morte, utilizou-se a mediana de SG para pacientes tratados com prednisona após quimioterapia (Tabela 1) (Fizazi et al., 2012).

\section{Medidas de efetividade}

A estimativa dos AVG propiciados por cada sequência de tratamento foi realizada por meio da relação entre os resultados de eficácia e probabilidades de transição de cada estado do modelo de Markov. O total de AVG a cada comparador ao final do tempo horizonte de 20 anos foi o resultado da soma dos AVG de cada ciclo de tratamento.

\section{Esquema terapêutico}

As posologias de abiraterona, enzalutamida e prednisona foram determinadas de acordo com os regimes utilizados nos estudos clínicos.
O regime de abiraterona consistiu em $1 \mathrm{~g}$ diário (quatro comprimidos de $250 \mathrm{mg}$ ) via oral, associado a $10 \mathrm{mg}$ de prednisona via oral (dois comprimidos de $5 \mathrm{mg}$ por dia) (de Bono et al., 2011; Ryan et al., 2013). A dose diária de enzalutamida foi de $160 \mathrm{mg}$ (quatro comprimidos de $40 \mathrm{mg}$ ) via oral (Beer et al., 2014; Scher et al., 2012). Para o docetaxel, o regime selecionado foi de $75 \mathrm{mg} / \mathrm{m}^{2}$ de superfície corpórea em 1 hora de infusão a cada 21 dias (Tannock et al., 2004). A superfície corpórea considerada foi de 1,89 $\mathrm{m}^{2}$.

\section{Uso de recursos e custos}

O uso de recursos estimados para cada um dos regimes terapêuticos foi realizado de acordo com as posologias descritas na seção "Esquema terapêutico" e está descrito na Tabela 2.

As taxas de ocorrência dos eventos adversos de grau 3 e acima foram extraídas dos estudos COU-AA-301, 302, PREVAIL, AFFIRM e de Tannock et al. e estão descritas na Tabela 3 (Beer et al., 2014; de Bono et al., 2011; Ryan et al., 2013; Scher et al., 2012; Tannock et al., 2004). Os recursos utilizados para o tratamento dos eventos adversos foram estimados com base na opinião de especialistas.

Os custos de procedimentos foram obtidos por meio da Classificação Brasileira Hierarquizada de Procedimentos Médicos (CBHPM, 2012). Os dados dos custos dos medicamentos foram extraídos da lista da Câmara de Regulação do Merca- 
Tabela 2. Recursos e custos dos regimes terapêuticos

\begin{tabular}{lccccc}
\hline Medicamento & Apresentação & Posologia & Quantidade mensal & Custo unitário & Custo mensal \\
\hline Abiraterona & Comprimido & $1 \mathrm{~g} /$ dia & 121,8 comprimidos & $R \$ 77,65$ & $R \$ 9.453,95$ \\
\hline Enzalutamida & Comprimido & $160 \mathrm{mg} /$ dia & 121,8 comprimidos & $R \$ 76,87$ & $R \$ 9.359,35$ \\
\hline Prednisona & Comprimido & $10 \mathrm{mg} /$ dia & 60,9 comprimidos & $R \$ 0,54$ & $R \$ 32,93$ \\
\hline Docetaxel & Ampola & $75 \mathrm{mg} / \mathrm{m}^{2} / 21$ dias & 1,4 ampola & $R \$ 7.578,46$ & $R \$ 10.984,26$ \\
\hline
\end{tabular}

Tabela 3. Frequência de eventos adversos por regime terapêutico em cada fase de tratamento

\begin{tabular}{|c|c|c|c|c|c|c|c|}
\hline \multirow[b]{2}{*}{ Evento adverso } & \multicolumn{3}{|c|}{ Tratamento pós-ADT } & \multirow{2}{*}{$\begin{array}{c}\text { Quimioterapia } \\
\text { Docetaxel }\end{array}$} & \multicolumn{3}{|c|}{ Tratamento pós-quimioterapia } \\
\hline & $\begin{array}{c}\text { Abiraterona + } \\
\text { Prednisona }\end{array}$ & Enzalutamida & Prednisona & & $\begin{array}{c}\text { Abiraterona + } \\
\text { Prednisona }\end{array}$ & Enzalutamida & $\begin{array}{l}\text { Prednisona } \\
\text { (cuidado } \\
\text { paliativo) }\end{array}$ \\
\hline Neutropenia & $0,0 \%$ & $0,0 \%$ & $0,0 \%$ & $32,0 \%$ & $0,0 \%$ & $0,0 \%$ & $0,0 \%$ \\
\hline Neutropenia febril & $0,0 \%$ & $0,0 \%$ & $0,0 \%$ & $3,0 \%$ & $0,0 \%$ & $0,0 \%$ & $0,0 \%$ \\
\hline Trombocitopenia & $0,0 \%$ & $0,0 \%$ & $0,0 \%$ & $1,0 \%$ & $1,4 \%$ & $1,0 \%$ & $0,5 \%$ \\
\hline Anemia & $0,0 \%$ & $0,0 \%$ & $0,0 \%$ & $5,0 \%$ & $7,8 \%$ & $7,8 \%$ & $8,1 \%$ \\
\hline Artralgia & $1,8 \%$ & $1,4 \%$ & $1,9 \%$ & $0,0 \%$ & $5,1 \%$ & $2,3 \%$ & $4,3 \%$ \\
\hline Astenia & $0,2 \%$ & $1,3 \%$ & $1,3 \%$ & $0,0 \%$ & $3,3 \%$ & $2,5 \%$ & $2,0 \%$ \\
\hline Diarreia & $1,1 \%$ & $0,2 \%$ & $0,9 \%$ & $32,0 \%$ & $1,1 \%$ & $1,1 \%$ & $1,3 \%$ \\
\hline Dispneia & $0,0 \%$ & $0,0 \%$ & $0,0 \%$ & $15,0 \%$ & $1,8 \%$ & $0,6 \%$ & $2,3 \%$ \\
\hline Fadiga & $2,4 \%$ & $1,8 \%$ & $1,9 \%$ & $5,0 \%$ & $9,1 \%$ & $6,3 \%$ & $10,4 \%$ \\
\hline Náusea & $0,0 \%$ & $0,0 \%$ & $0,0 \%$ & $42,0 \%$ & $2,1 \%$ & $1,5 \%$ & $2,8 \%$ \\
\hline Vômito & $0,0 \%$ & $0,0 \%$ & $0,0 \%$ & $0,0 \%$ & $2,7 \%$ & $1,1 \%$ & $3,0 \%$ \\
\hline Edema & $0,9 \%$ & $0,2 \%$ & $1,7 \%$ & $19,0 \%$ & $2,5 \%$ & $1,0 \%$ & $1,0 \%$ \\
\hline Hipocalemia & $0,0 \%$ & $0,0 \%$ & $0,0 \%$ & $0,0 \%$ & $0,0 \%$ & $0,0 \%$ & $0,0 \%$ \\
\hline Hipertensão & $4,2 \%$ & $6,8 \%$ & $3,1 \%$ & $0,0 \%$ & $1,3 \%$ & $2,0 \%$ & $0,3 \%$ \\
\hline Dor nas costas & $2,8 \%$ & $2,5 \%$ & $3,9 \%$ & $0,0 \%$ & $7,1 \%$ & $5,0 \%$ & $10,2 \%$ \\
\hline Neuropatia & $0,0 \%$ & $0,0 \%$ & $0,0 \%$ & $0,0 \%$ & $0,0 \%$ & $0,0 \%$ & $0,0 \%$ \\
\hline
\end{tabular}

ADT: terapia de privação androgênica.

do de Medicamentos (CMED) atualizada em janeiro de 2016, considerando preço de fábrica 18\%. Os custos unitários dos medicamentos foram de $\mathrm{R} \$ 77,65$ por comprimido de abiraterona, $\mathrm{R} \$ 76,87$ por comprimido de enzalutamida, $\mathrm{R} \$ 0,54$ por comprimido de prednisona e $\mathrm{R} \$ 3.789,23$ por ampola de docetaxel (CMED, 2016).

O custo de administração de docetaxel a cada 21 dias foi estimado com base no uso de recursos de uma clínica de oncologia no Brasil e considerou os custos de honorários, materiais e taxa de sala (CBHPM, 2012; SIMPRO, 2011). O custo total de administração foi de $\mathrm{R} \$ 337,19$. Os demais regimes não possuem custos de administração por serem medicamentos orais.

Os recursos utilizados no estado de monitoramento foram consultas com oncologista e procedimentos de monitorização, cuja frequência foi estimada com base nas diretrizes de tratamento do câncer de próstata da Associação Europeia de Urologia, nos estudos clínicos e nas bulas dos medica- mentos (de Bono et al., 2011; Heidenreich, 2012; Tannock et al., 2004). Essas estimativas foram validadas por um oncologista.

Adotou-se uma taxa de desconto de $5 \%$ ao ano para os custos e benefícios utilizados no modelo, conforme recomendado pela Diretriz Metodológica de Avaliação Econômica brasileira (Brasil, 2014).

\section{Análise de sensibilidade univariada}

Para determinar a robustez do resultado e os parâmetros de maior impacto nas variáveis de custo e efetividade do modelo, uma análise de sensibilidade univariada foi conduzida com os seguintes parâmetros: taxa de desconto (0\% e 10\%); preço de abiraterona ( $\pm 10 \%)$; custo dos eventos adversos $( \pm 20 \%)$; duração do tratamento com abiraterona pós-quimioterapia $( \pm 20 \%) ;$ sobrevida global do tratamento com abiraterona pós-quimioterapia ( $\pm 20 \%)$; análise sem ajuste das curvas de eficácia dos tratamentos com abiraterona ou enzalutamida no pós-ADT. 


\section{Resultados}

O tempo de tratamento com a sequência AP-DOC-ENZ é menor do que com a sequência ENZ-DOC-AP. Para AP-DOC-ENZ, o tempo médio de tratamento pós-ADT é de 13,73 meses, seguidos de 5,54 meses de quimioterapia e 3,41 meses de tratamento pós-quimioterapia. Já para ENZ-DOC-AP, o tempo de tratamento para esses três estados é de 15,11, 5,78 e 3,15 meses, respectivamente (Figura 2).

Os custos com AP-DOC-ENZ foram menores nos estados de tratamento pós-terapia de privação androgênica, quimioterapia, cuidado paliativo e morte (Figura 3). O estado que apresentou maior impacto no custo total do tratamento

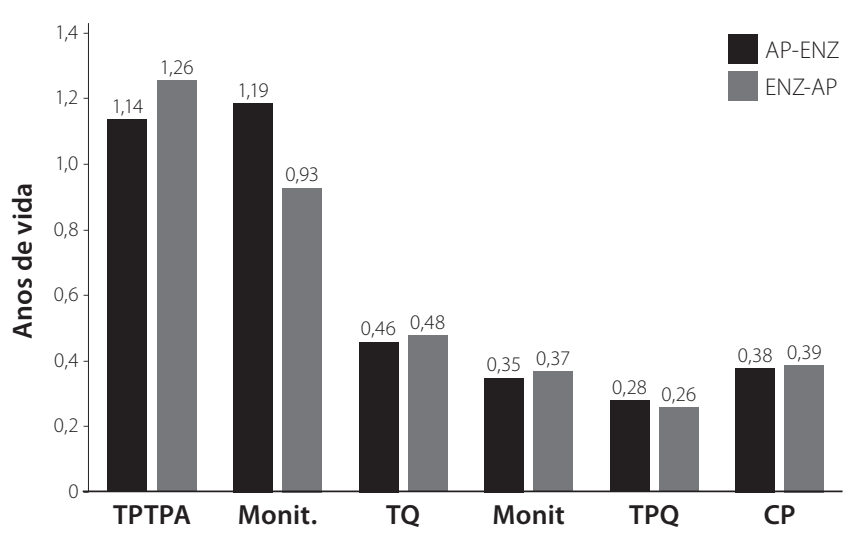

AP-ENZ: abiraterona associada à prednisona pós-ADT e enzalutamida pós-quimioterapia; ENZ-AP: enzalutamida pós-ADT e abiraterona associada à prednisona pós-quimioterapia; TPTPA: tratamento pós-terapia de privação androgênica; Monit.: monitoramento; TQ: tratamento quimioterápico; TPQ: tratamento pós-quimioterapia; CP: cuidados paliativos.

Figura 2. Tempo gasto por fase de tratamento. foi o estado pós-ADT (48\% para a sequência AP-DOC-ENZ e 50\% para ENZ-DOC-AP), e o custo total do estado foi de $\mathrm{R} \$ 125.560,00$ para abiraterona e de $\mathrm{R} \$ 136.086,00$ para enzalutamida, o que representa uma redução de 7,3\%. Essa redução foi de 4,3\% para o estado de quimioterapia, de 3,4\% para cuidado paliativo e de $0,5 \%$ para o estado de morte.

A sequência AP-DOC-ENZ apresentou maior número de AVG (3,37 versus 3,28 para ENZ-DOC-AP) (Tabela 4). Apresentou também menor custo total do que a sequência oposta, totalizando $\mathrm{R} \$ 262.801,00$ versus $\mathrm{R} \$ 274.165,00$ da sequência oposta, portanto foi considerada dominante em relação à sequência comparadora (Tabela 4).

$\mathrm{Na}$ análise de sensibilidade univariada, o modelo mostrou-se mais sensível à variável de preço da abiraterona. Entretanto, mesmo com o aumento de até $10 \%$ no preço, ainda mantiveram a sequência AP-DOC-ENZ como de menor custo. Apesar de a diferença de efetividade ser discreta, ela permaneceu positiva em todos os cenários avaliados (Figuras 4 e 5).

Tabela 4. Resultados

\begin{tabular}{lcccc}
\hline & \multicolumn{2}{c}{ Sem desconto } & \multicolumn{2}{c}{ Desconto de 5\% } \\
\cline { 2 - 5 } Comparador & Custos & AVG & Custos & AVG \\
\hline AP-ENZ & $R \$ 293.117,27$ & 3,81 & $R \$ 262.800,82$ & 3,37 \\
\hline ENZ-AP & $R \$ 304.644,35$ & 3,69 & $R \$ 274.164,59$ & 3,28
\end{tabular}

AP-ENZ: abiraterona associada à prednisona pós-ADT e enzalutamida pós-quimioterapia; ENZ-AP: enzalutamida pós-terapia de privação androgênica e abiraterona associada à prednisona pós-quimioterapia; AVG: anos de vida ganhos.

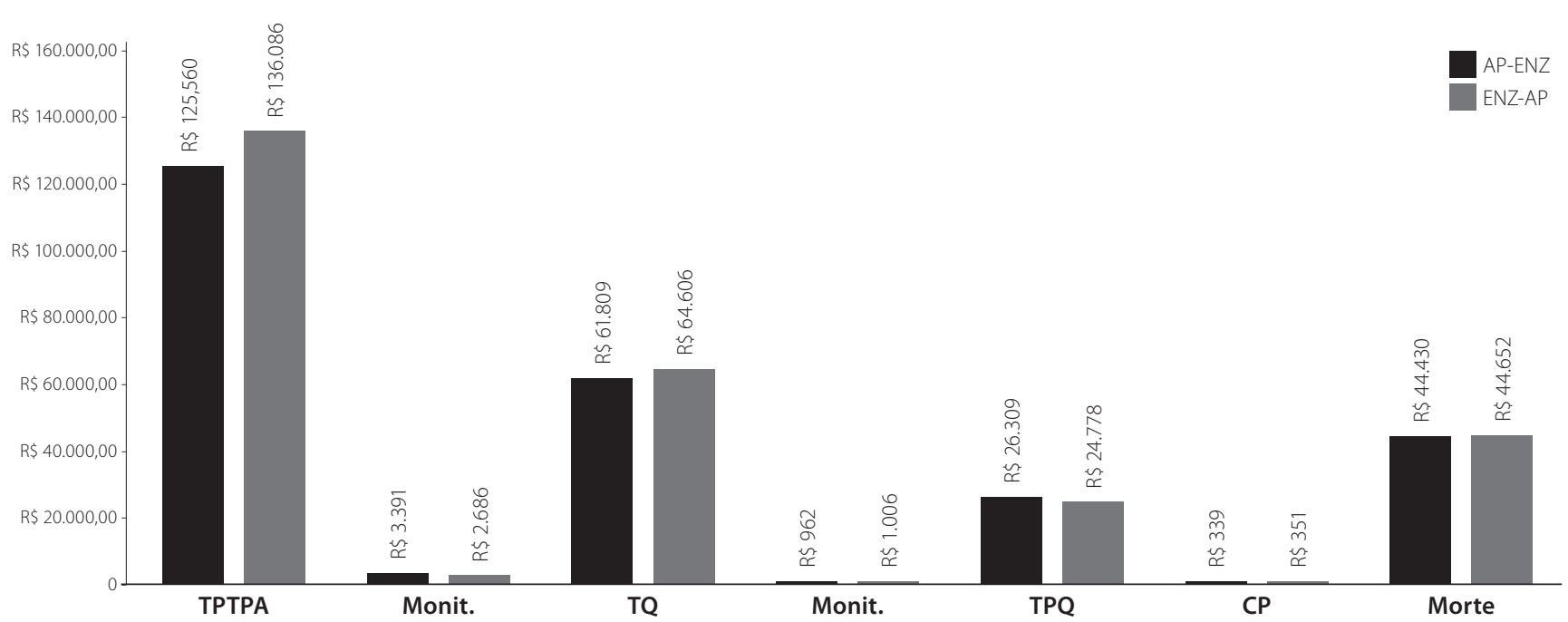

AP-ENZ: abiraterona associada à prednisona pós-ADT e enzalutamida pós-quimioterapia; ENZ-AP: enzalutamida pós-ADT e abiraterona associada à prednisona pós-quimioterapia; TPTPA: tratamento pós-terapia de privação androgênica; Monit.: monitoramento; TQ: tratamento quimioterápico; TPQ: tratamento pós-quimioterapia; CP: cuidados paliativos.

Figura 3. Custo por fase de tratamento. 


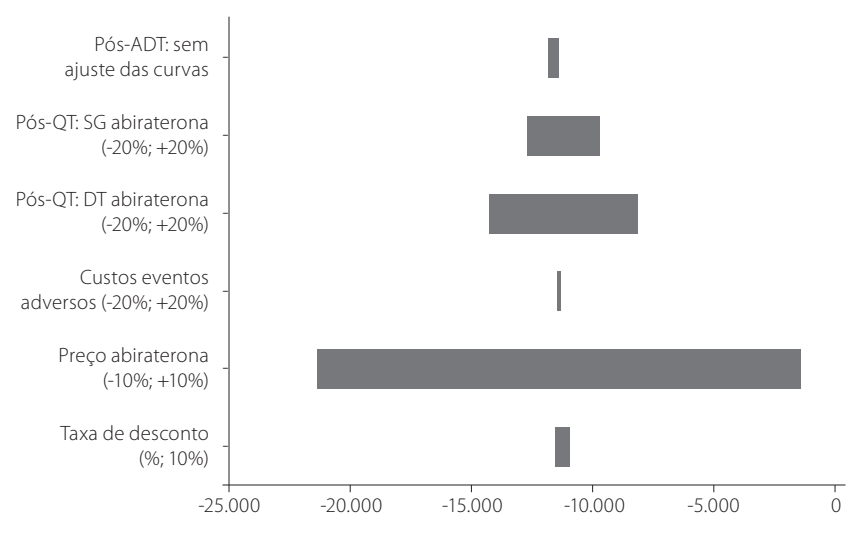

SG: sobrevida global; DT: duração do tratamento.

Figura 4. Diagrama de tornado: variável custo (análise de sensibilidade univariada).

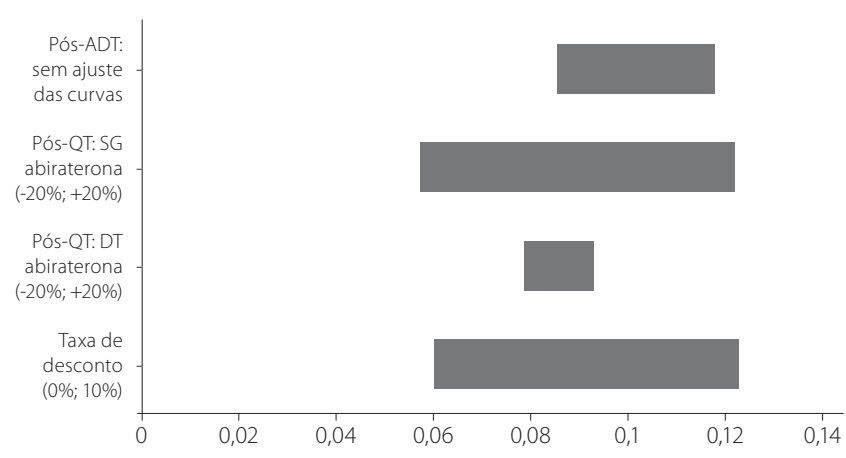

SG: sobrevida global; DT: duração do tratamento.

Figura 5. Diagrama de tornado: variável efetividade (análise de sensibilidade univariada).

\section{Discussão}

Este modelo de custo-efetividade foi estruturado para refletir a prática clínica do cuidado de pacientes com CPRC. Após a falha da terapia de privação androgênica, demonstrou-se que a adoção da sequência AP-DOC-ENZ é dominante em relação à sequência ENZ-DOC-AP, uma vez que apresentou custos totais de tratamento menores e maior efetividade, mensurada pela quantidade de AVG.

Notou-se que os principais direcionadores do resultado foram o tempo de tratamento (para custos) e as diferenças de sobrevida (para efetividade) entre o uso de cada terapia, especialmente na indicação pós-ADT. A redução do tempo de tratamento diminui os custos com os medicamentos e o período no qual o paciente é exposto a eventos adversos, cujo manejo também impacta no custo total de tratamento.

O modelo desenvolvido simula a realidade clínica de um paciente com CPRC, e a análise de custo-efetividade reuniu todos os parâmetros relacionados aos tratamentos com sequências comparadoras. Por tratar-se de uma simulação, essa análise possui algumas limitações. Devido à inexistência de estudos de comparação direta entre as sequências AP-DOC-ENZ e ENZ-DOC-AP no tratamento de CPRC, foram utilizados dados de estudos particulares de cada medicamento comparando-os pelo método de MAIC. Apesar de esse método reduzir as diferenças entre os estudos clínicos, fatores não observáveis nos dados reportados podem ser um fator de influência no resultado, como diferenças entre os grupos placebo ou fatores que influenciem apenas os grupos tratamento e não placebo (Signorovitch, 2012). Ainda, a sobrevida global do estado de cuidado paliativo foi assumida como igual à do grupo placebo mais prednisona do COU-AA-301 (Fizazi et al., 2012). Essa definição confere um nível de incerteza para os pacientes que se encontram nesse estado, uma vez que não foram encontrados dados específicos sobre eles. $\mathrm{O}$ impacto dessa limitação nos resultados da análise não pode ser quantificado, visto que ele pode tanto subestimar quanto superestimar os resultados do modelo. Importante ressaltar que, na análise de sensibilidade univariada, se verificou que utilizar os parâmetros dos estudos sem levar em consideração o método de comparação indireta adotado faz com que as diferenças se intensifiquem: a sequência AP-DOC-ENZ passa a ser mais econômica e de maior eficácia que a sequência oposta.

Um estudo observacional retrospectivo realizado com pacientes nos Estados Unidos relatou que, comparado ao uso da enzalutamida, o uso da abiraterona pode estar relacionado à redução de fadiga, fraqueza e eventos adversos no sistema nervoso central, como amnésia, ansiedade, disfunções cognitivas, convulsões, distúrbios de atenção, alucinações e insônia, por exemplo. Essa associação também foi realizada com a diminuição da ocorrência de eventos adversos que acarretassem a redução das doses desses medicamentos (Kane, 2016).

Com o desenvolvimento de novas tecnologias para o tratamento de CPRC, como a abiraterona e a enzalutamida, a melhor forma de sequenciar os medicamentos disponíveis tornou-se a principal dificuldade.

As evidências disponíveis na literatura, ainda que limitadas, apontam para uma menor eficácia dos novos agentes em pacientes previamente tratados com eles. Um estudo observacional retrospectivo multicêntrico analisou a eficácia de enzalutamida após a falha de abiraterona e docetaxel em 61 pacientes. Houve redução maior ou igual a 30\% de antígeno prostático específico (PSA) em 28\% dos pacientes e maior ou igual a 50\% em 15\% deles. A sobrevida livre de progressão (SLP) foi de 12 semanas (Badrising et al., 2014). Outro estudo retrospectivo realizado com 39 pacientes com CPRC verificou que $41 \%$ dos pacientes apresentaram queda de PSA maior ou igual a 30\%. Essa redução foi maior ou igual a 50\% em 12,8\% e maior ou igual a $90 \%$ em 2,5\% dos pacientes (Bianchini et al., 2014). Ainda sobre o uso da enzalutamida, um estudo observacional investigou a sua eficácia pós-abiraterona e 
pós-docetaxel. Não houve diferenças estatisticamente significativas na queda de PSA, taxa de resposta e SLP entre os grupos (Suzman et al., 2014). Noonan et al. analisaram retrospectivamente uma coorte de 30 pacientes com CPRC que receberam abiraterona após a falha de docetaxel e enzalutamida. A queda de PSA maior ou igual a 30\% foi observada em $10 \%$ dos pacientes e maior ou igual a $50 \%$ em $3 \%$ deles (Noonan et al., 2013). Esses estudos oferecem dados de mundo real sobre o uso de algumas das possíveis sequências de tratamento de CPRC; porém, trata-se de análises retrospectivas com coortes pequenas de pacientes em um mesmo país e que estão sujeitas a extensas limitações.

Portanto, uma importante limitação do estudo é a ausência de ensaios clínicos randomizados com dados de eficácia na indicação pós-quimioterapia de pacientes previamente expostos, após a falha da terapia de privação androgênica, ao acetato de abiraterona ou enzalutamida. Não é possível determinar se essa limitação subestima ou superestima os resultados de custo e efetividade do modelo. Entretanto, é importante notar que na análise de sensibilidade univariada verificou-se que os parâmetros de duração do tratamento e sobrevida global nos pacientes pós-quimioterapia apresentam baixo impacto no resultado final.

Quanto às evidências sobre a eficácia das sequências terapêuticas, um estudo de coorte retrospectiva comparou o uso sequencial de abiraterona e enzalutamida dos pacientes tratados no Sidney Kimmel Comprehensive Cancer Center at Johns Hopkins University (Maughan, 2017). Apesar de a intervenção não ser a mesma (o estudo não considera o uso de quimioterapia com docetaxel entre os agentes hormonais), o resultado da análise de sobrevida global está em linha com os achados do modelo. Após regressão multivariada, o hazard ratio das curvas de sobrevida global entre os grupos ficou próxima de ser estatisticamente significativa ( $H R$ 0,57, $95 \%$ IC 0,29-1,11, $p=0,098$ ).

Uma análise de custo por evento evitado (COPE) comparando a enzalutamida com a abiraterona associada à prednisona para o tratamento de CPRC no Sistema de Saúde Suplementar brasileiro foi publicada por Stefani et al. e relatou que o custo para evitar uma morte é menor com a enzalutamida. Entretanto, os resultados apresentados pela presente análise de custo-efetividade permitem conclusões mais precisas sobre o uso dessas duas estratégias terapêuticas, uma vez que a análise COPE foi exposta a limitações críticas. Na COPE não foi utilizado nenhum método que permitisse a comparação dos resultados dos estudos COU-AA-302 e AFFIRM. Além disso, essa análise referiu-se apenas ao uso de abiraterona e enzalutamida após o regime quimioterápico com docetaxel, desconsiderando as estratégias de sequenciamento, e não foram consideradas as ocorrências de eventos adversos, o que representa parte importante dos custos relacionados a ambos os tratamentos (Stefani et al., 2015). Por fim, a própria conclusão de que o custo para evitar uma morte é menor não pode ser feita com base nos resultados da análise, devido à larga amplitude dos intervalos de confiança dos resultados individuais das terapias (Trufelli, 2016).

Por fim, o preço de aquisição dos novos agentes (abiraterona e enzalutamida) é a principal variável que afeta os resultados de custo do modelo. $\mathrm{Na}$ análise de sensibilidade univariada, mesmo um custo de aquisição de abiraterona até 11\% maior que o de enzalutamida ainda mantém a sequência de tratamento AP-DOC-ENZ como a de menor custo. Esse modelo toma como base para custo o preço lista de cada um dos medicamentos, mas na prática esse é o preço máximo a ser praticado no mercado, podendo, portanto, sofrer variações.

Futuros estudos que comparem diretamente a eficácia e segurança das diferentes sequências terapêuticas poderão ser uma fonte de dados que permitam uma avaliação econômica mais precisa sobre as suas aplicações. Entretanto, levando em consideração os dados de sobrevida dos medicamentos, o tempo de tratamento e a ocorrência de eventos adversos, a presente análise indicou uma melhor razão de custo-efetividade incremental para o uso da sequência AP-DOC-ENZ no sistema de saúde suplementar brasileiro.

\section{Conclusão}

O uso da sequência AP-DOC-ENZ foi associado a melhores resultados de custo-efetividade e apresentou-se dominante ao uso da sequência ENZ-DOC-AP no tratamento de CPRC, embora o resultado do ganho de efetividade tenha se mostrado pequeno. Esse resultado indica que a adoção da sequência AP-DOC-ENZ pode aumentar a sobrevida dos pacientes e diminuir o custo total de tratamento no Sistema de Saúde Suplementar brasileiro.

\section{Agradecimentos}

Agradecemos a Evidências - Kantar Health pelo desenvolvimento do medical writing e a escrita deste artigo.

\section{Referências bibliográficas}

Attard G, Belldegrun AS, de Bono JS. Selective blockade of androgenic steroid synthesis by novel lyase inhibitors as a therapeutic strategy for treating metastatic prostate cancer. BJU Int. 2005;96(9):1241-6.

Badrising S, van der Noort V, van Oort IM, van den Berg HP, Los M, Hamberg P, et al. Clinical activity and tolerability of enzalutamide (MDV3100) in patients with metastatic, castration-resistant prostate cancer who progress after docetaxel and abiraterone treatment. Cancer. 2014;120(7):968-75.

Beer TM, Armstrong AJ, Rathkopf DE, Loriot Y, Sternberg CN, Higano CS, et al.; PREVAIL Investigators. Enzalutamide in metastatic prostate cancer before chemotherapy. N Engl J Med. 2014;371(5):424-33. 
Beltran H, Beer TM, Carducci MA, de Bono J, Gleave M, Hussain M, et al. New therapies for castration-resistant prostate cancer: efficacy and safety. Eur Urol. 2011;60(2):279-90.

Bianchini D, Lorente D, Rodriguez-Vida A, Omlin A, Pezaro C, Ferraldeschi R, et al. Antitumour activity of enzalutamide (MDV3100) in patients with metastatic castration-resistant prostate cancer (CRPC) pre-treated with docetaxel and abiraterone. Eur J Cancer. 2014;50(1):78-84.

Bianchini D, Zivi A, Sandhu S, de Bono JS. Horizon scanning for novel therapeutics for the treatment of prostate cancer. Ann Oncol. 2010;21 Suppl 7:vii43-55.

Botrel TE, Clark O, Pompeo AC, Bretas FF, Sadi MV, Ferreira U, et al. Immunotherapy with Sipuleucel-T (APC8015) in patients with metastatic castration-refractory prostate cancer (mCRPC): a systematic review and meta-analysis. Int Braz J Urol. 2012;38(6):717-27.

Brasil. Ministério da Saúde, Secretaria de Ciência, Tecnologia e Insumos Estratégicos, Departamento de Ciência e Tecnologia. Diretrizes Metodológicas: Diretriz de Avaliação Econômica. 2ª ed. 2014. Disponível em: < http://bvsms.saude.gov.br/bvs/publicacoes/diretrizes_ metodologicas_diretriz_avaliacao_economica.pdf>.

Cardoso Filho FA, organizador. CBHPM - Classificação Brasileira Hierarquizada de Procedimentos Médicos. São Paulo: Associação Médica Brasileira; 2012.

Cha E, Fong L. Therapeutic vaccines for prostate cancer. Curr Opin Mol Ther. 2010;12(1):77-85.

Chang SS, Kibel AS. The role of systemic cytotoxic therapy for prostate cancer. BJU Int. 2009;103(1):8-17.

Câmara de Regulação do Mercado de Medicamentos (CMED). Lista de preços e medicamentos: preço fábrica e preço máximo de venda ao governo. Agência Nacional de Vigilância Sanitária. Secretaria Executiva. 2016. Atualização: janeiro de 2016.

de Bono JS, Logothetis CJ, Molina A, Fizazi K, North S, Chu L, et al.; COUAA-301 Investigators. Abiraterone and increased survival in metastatic prostate cancer. N Engl J Med. 2011;364(21):1995-2005.

Dearden L, Majer I, van de Wetering G. Acost comparison of treatment with abirateroneacetate plus prednisone in the pre-chemotherapy setting followed by enzalutamide in the post-chemotherapy setting versus the opposite treatment sequence in metastatic castration resistant prostate cancer patients with non-visceral metastases. ISPOR 18th Annual European Congress; 2015.

Fizazi K, Scher HI, Molina A, Logothetis CJ, Chi KN, Jones RJ, et al.; COUAA-301 Investigators. Abiraterone acetate for treatment of metastatic castration-resistant prostate cancer: final overall survival analysis of the COU-AA-301 randomised, double-blind, placebo-controlled phase 3 study. Lancet Oncol. 2012;13(10):983-92.

Heidenreich ABP, Bellmunt J, et al. Guidelines on Prostate Cancer. European Association of Urology. 2012.

Inca. Estimativa 2016: incidência de câncer no Brasil. 2015.

Kane DPASBLGBEYXPLCJ. Assessment of Central Nervous System (CNS) and Dose Reduction Events in Patients Treated With Abiraterone Acetate Plus Prednisone (AA+P) or Enzalutamide (ENZ). ASCO Annual Meeting; Chicago, IL, USA; 2016.

Maughan BL, Luber B, Nadal R, Antonarakis ES. Comparing Sequencing of Abiraterone and Enzalutamide in Men With Metastatic CastrationResistant Prostate Cancer: A Retrospective Study. Prostate. 2017;77(1):33-40.

Noonan KL, North S, Bitting RL, Armstrong AJ, Ellard SL, Chi KN. Clinical activity of abiraterone acetate in patients with metastatic castrationresistant prostate cancer progressing after enzalutamide. Ann Oncol. 2013;24(7):1802-7.
Petrylak DP, Tangen CM, Hussain MH, Lara PN Jr, Jones JA, Taplin ME, et al. Docetaxel and estramustine compared with mitoxantrone and prednisone for advanced refractory prostate cancer. N Engl J Med. 2004;351(15):1513-20.

Ryan CJ, Smith MR, de Bono JS, Molina A, Logothetis CJ, de Souza P, et al.; COU-AA-302 Investigators. Abiraterone in metastatic prostate cancer without previous chemotherapy. N Engl J Med. 2013;368(2):138-48.

Ryan CJ, Smith MR, Fizazi K, Saad F, Mulders PF, Sternberg CN, et al.; COUAA-302 Investigators. Abiraterone acetate plus prednisone versus placebo plus prednisone in chemotherapy-naive men with metastatic castration-resistant prostate cancer (COU-AA-302): final overall survival analysis of a randomised, double-blind, placebo-controlled phase 3 study. Lancet Oncol. 2015;16(2):152-60.

Sadi MV, Ferreira U, Clark O, et al. Câncer da próstata resistente à castração (CPRC). 25a Reunião do Comitê Brasileiro de Estudos em Uro-Oncologia (CoBEU) em São Paulo; 2011.

Scher HI, Fizazi K, Saad F, Taplin ME, Sternberg CN, Miller K, et al.; AFFIRM Investigators. Increased survival with enzalutamide in prostate cancer after chemotherapy. N Engl J Med. 2012;367(13):1187-97.

Scher HI, Heller G, Molina A, Kheoh TS, Attard G, Moreira J, et al. Evaluation of circulating tumor cell (CTC) enumeration as an efficacy response biomarker of overall survival (OS) in metastatic castration-resistant prostate cancer (MCRPC): Planned final analysis (FA) of COU-AA-301, a randomized double-blind, placebo-controlled phase III study of abiraterone acetate (AA) plus low-dose prednisone (P) post docetaxel. J Clin Oncol 2011;29 Suppl (Abstr LBA4517).

Signorovitch JE, Sikirica V, Erder MH, Xie J, Lu M, Hodgkins PS, et al. Matchingadjusted indirect comparisons: a new tool for timely comparative effectiveness research. Value Health. 2012;15(6):940-7.

SIMPRO R. SIMPRO. Informações e Soluções em Saúde. 2011.

Stefani S TV, Fay A, Abadi M, Flanders S, Bul C, Sasse A. Custo por evento evitado da enzalutamida versus abiraterona associada à prednisona em homens com câncer de próstata resistente à castração metastático, sob a perspectiva do Sistema de Saúde Suplementar no Brasil. J Bras Econ Saúde. 2015;7(3):127-37.

Suzman DL, Luber B, Schweizer MT, Nadal R, Antonarakis ES. Clinical activity of enzalutamide versus docetaxel in men with castrationresistant prostate cancer progressing after abiraterone. Prostate. 2014;74(13):1278-85.

Tannock IF, de Wit R, Berry WR, Horti J, Pluzanska A, Chi KN, et al.; TAX 327 Investigator. Docetaxel plus prednisone or mitoxantrone plus prednisone for advanced prostate cancer. N Engl J Med. 2004;351(15):1502-12.

Tannock IF, Osoba D, Stockler MR, Ernst DS, Neville AJ, Moore MJ, et al. Chemotherapy with mitoxantrone plus prednisone or prednisone alone for symptomatic hormone-resistant prostate cancer: a Canadian randomized trial with palliative end points. J Clin Oncol. 1996;14(6):1756-64.

Trufelli D, Pititto L, Asano E, Martins E. Carta ao editor. J Bras Econ Saúde. 2016;8(2):172.

Tunn U. The current status of intermittent androgen deprivation (IAD) therapy for prostate cancer: putting IAD under the spotlight. BJU Int. 2007;99 Suppl 1:19-22. 